MATEC Web of Conferences 44, 01085 (2016)

DOI: $10.1051 /$ matecconf/20164401085

(c) Owned by the authors, published by EDP Sciences, 2016

\title{
Research on routing algorithm based on the VANET
}

\author{
Li AN ${ }^{1}$, Yong Mei ZHAO ${ }^{1}$, Peng XIE and Qiong WANG ${ }^{1}$ \\ Science College of Air Force Engineering University
}

\begin{abstract}
For the characteristics of high speed mobility of nodes, frequent changes of dynamic topology and frequent interrupts of the communication links in the VANET, this paper analyzed the defect of the current mobile ad-hoc network routing protocol, and carried on the simulation analysis on the adaptability of AODV, DSR and DSDV routing protocols in VANET applications in the VANET. Through the above research, this paper obtained the conclusion that the AODV routing protocol is more suitable for vehicular ad hoc network environment
\end{abstract}

\section{INTRODUCTION}

In recent years, on the account of the rapid development of wireless communication technology and the urgent need in the information technology of transportation field, the VANET(vehicular Ad Hoc Networks), one important foundation concept of intelligent transportation systems, was spawned. VANET is a type of special mobile ad hoc network, which creatively applies Ad hoc network in inter-vehicle communications, allows the drivers to obtain status information of other vehicles over the horizon (such as speed, direction, position, pressure of brakes plate, etc.) and real-time traffic information. Its main characteristic is the network nodes can move arbitrarily, frequent changes in network topology, and each node has the function of both host and router. Besides, VANET does not need the support of fixed infrastructure, can quickly set up a mobile communication network at any time and any place, which can not only increase the transportation efficiency, also the other facilities such as the safety and the reliability. Due to the high mobility of VANET, the unpredictable nature of road and the uncertainty of driving behavior, it is difficult to provide effective routing mechanism in such a complex situation.

VANET applications are very extensive, in civil in daily life or military, such as transport fleet, combat command team and the most popular of the "self driving tour" nowadays, which can solve the inter communication of the whole vehicles, also can ensure the consistency about getting the

Corresponding author: ghxgbx@163.com important information in the march of the whole vehicles.

For the characteristics of high speed mobility of nodes, frequent changes of dynamic topology and frequent interrupts of the communication links in the VANET, this paper analyzed whether the current routing protocol of mobile ad hoc network can meet the communication requirements of VANET or not.

\section{OVERVIEW OF ROUTING PROTOCOLS IN THE MOBILE AD HOC NETWORKS}

The function of routing is implemented in the network layer, which is mainly to specify the path from the source node to the destination node for the packet transmission data in the communication system. Mobile ad hoc routing protocol for inter vehicles packet transmission data is used to provide path information $\mathrm{t}$. It is that the main goal is to meet practical need, at the same time to minimize the network overhead and achieve the maximization of overall utilization of resources, improve the network throughput.

The mobile ad hoc routing protocol has been widely studied. And it has different classification based on different standards. According to the routing strategy to divide, it is mainly divided into the proactive routing protocol and the on-demand routing protocol. The proactive routing protocol requires that each node should maintain a routing table that contains the routing information of all other nodes in real time, also known as the table driven routing protocol. 
For example, the Destination-Sequenced Distance-Vector Routing protocol ( DSDV), regardless of whether the nodes need to send data or not, regularly broadcast updates. In an environment with large scale and fast topology changes, the continuous updating information can lead to excessive channel occupancy, which greatly reduces the performance of the system. The On-demand routing protocols is on the basis of data transmission request, passively searches routes from the source node to the destination node. If there is no data transmission request, it does not need to exchange routing information, also known as passive routing protocols. For example, the Ad hoc On-demand Distance Vector routing (AODV )protocol, is improved on the basis of the DSDV protocol, a combination of DSDV protocol and Dynamic Source Routing(DSR) protocol, broadcasts routing information only when it is needed, which reduces the total amount of broadcast. The on-demand routing protocol is a special type of routing protocols in the mobile ad hoc networks, which has the advantage of reducing overhead and improving network throughput. However, whether the route can reach the destination node each time and the delay of the route establishment can not be certain. For vehicular ad hoc networks, it is very difficult to maintain the routing table in real time, and the on-demand routing protocol is more suitable for vehicular ad hoc networks.

\section{THE ANALYSIS ON VEHICLES MODEL}

Considering the influence of road environment and traffic facilities, and the limitation of the safety distance between the vehicles, the simulation model of vehicular ad hoc network is different from the general model of mobile ad hoc network, and has its own unique characteristics.

Based on the analysis of vehicular ad hoc network routing protocol, we choose the AODV routing protocol which has been used widely as the protocol model. While needed to be improved in safety, the AODV routing protocol is also not entirely designed in

$$
\text { average end }- \text { to }- \text { end delay }=\frac{\text { the sum of the packets received delay of the destination node }}{\text { the number of packets received by the destination node }}
$$

In this paper, 20 mobile nodes made up the vehicular ad hoc network, with the node speed maintained uniform $18 \mathrm{~m} / \mathrm{s}$ and the source node packet rate set to 3 packets $/ \mathrm{s}$. In the scene of 5 , accordance with the characteristics of vehicular ad hoc networks and is not the most suitable protocols either, but the AODV Routing Protocol is able to quickly adapt to the dynamic changes of network topology. At the same time, the storage overhead and network utilization of the AODV Routing Protocol are relatively small. In addition, the vehicular ad hoc networks have a symmetrical bidirectional connectivity. For instance, there are two adjacent vehicle nodes $\mathrm{A}$ and $\mathrm{B}$. If $\mathrm{A}$ can monitor the communication content of $\mathrm{B}$, then $\mathrm{B}$ can also monitor the communication content of A. So this paper chose the AODV routing protocol as the routing protocol model. It could be verified by simulation: The speed of each vehicle node was stable, and the structure of the linear vehicles would not change. The free communication between adjacent two nodes could be guaranteed under normal operating conditions, and the communication link would not be interrupted.

\section{THE SIMULATION EVALUATION OF VEHICLES MODEL PERFORMANCE}

There are several key indicators to measure the advantages and disadvantages of a VANET routing protocol performance, which include throughput, average end-to-end delay, average packet transmission rate, routing overhead, routing request time and energy consumption etc. In order to evaluate the applicability of AODV, DSR and DSDV routing protocols in VANET, this paper set the number of different communication source nodes, did the simulation contrast experiments on the average delay of end to end, the loss rate of packet and other indicators, to determine which kind of routing protocol is the most suitable for linear vehicles model.

\subsection{Average end-to-end delay}

Average end to end delay for data packets is the sum of all the possible delays, such as cache delay, interface queue delay, MAC layer retransmission delay, packet air propagation delay, and transformation time. The expression looks like the following:
10, 15 and 20 communication source nodes, through the simulation contrast experiment, the average end-to-end delay performance was obtained as shown in Table 1 and Figure 1 
Table 1. the average end-to-end delay performanceof AODV、DSR、DSDV

\begin{tabular}{|c|c|c|c|c|c|c|c|c|c|c|c|c|c|c|c|}
\hline & \multicolumn{3}{|c|}{$50 \mathrm{~s}$} & \multicolumn{3}{|c|}{$100 \mathrm{~s}$} & \multicolumn{3}{|c|}{$200 \mathrm{~s}$} & \multicolumn{3}{|c|}{$400 \mathrm{~s}$} & \multicolumn{3}{|c|}{$600 \mathrm{~s}$} \\
\hline & AODV & DSR & DSDV & $\mathrm{AODV}$ & DSR & DSDV & AODV & DSR & DSDV & AODV & $\mathrm{V}$ DSR & DSDV & AODV & $V$ DSR & DSDV \\
\hline 5 & 0.065 & 0.039 & 0 & 0.060 & 0.039 & 0 & 0.057 & 0.038 & 0.041 & 0.056 & 50.038 & 0.050 & 0.056 & 0.038 & 0.053 \\
\hline 10 & 0.071 & 0.035 & 0 & 0.060 & 0.033 & 0.029 & 0.077 & 0.035 & 0.026 & 0.062 & 0.030 & 0.040 & 0.057 & 0.029 & 0.043 \\
\hline 15 & 0.071 & 0.035 & 0 & 0.060 & 0.033 & 0.020 & 0.088 & 0.028 & 0.020 & 0.059 & 0.023 & 0.027 & 0.064 & 0.022 & 0.027 \\
\hline 20 & 0.071 & 0.035 & 0 & 0.058 & 0.030 & 0.005 & 0.094 & 0.021 & 0.016 & 0.067 & 70.019 & 0.024 & 0.059 & 0.018 & 0.031 \\
\hline
\end{tabular}

As could be seen from Figure 1.1, with the increase of the pause time, the average end-toend delay of AODV and DSR routing protocols had different degrees of decline, while the average end to end delay of DSDV routing protocol had a certain degree of increase. But with the increase of the communication link and the number of source nodes, the three types of protocols showed different trends. Further analysis could be concluded as follows: In the case of short pause time, the average end-to-end delay of DSDV was the smallest. The average end-toend delay of DSR was the more and the average end-to-end delay of AODV was the maximum. With the extension of the pause time, the three routing protocols showed
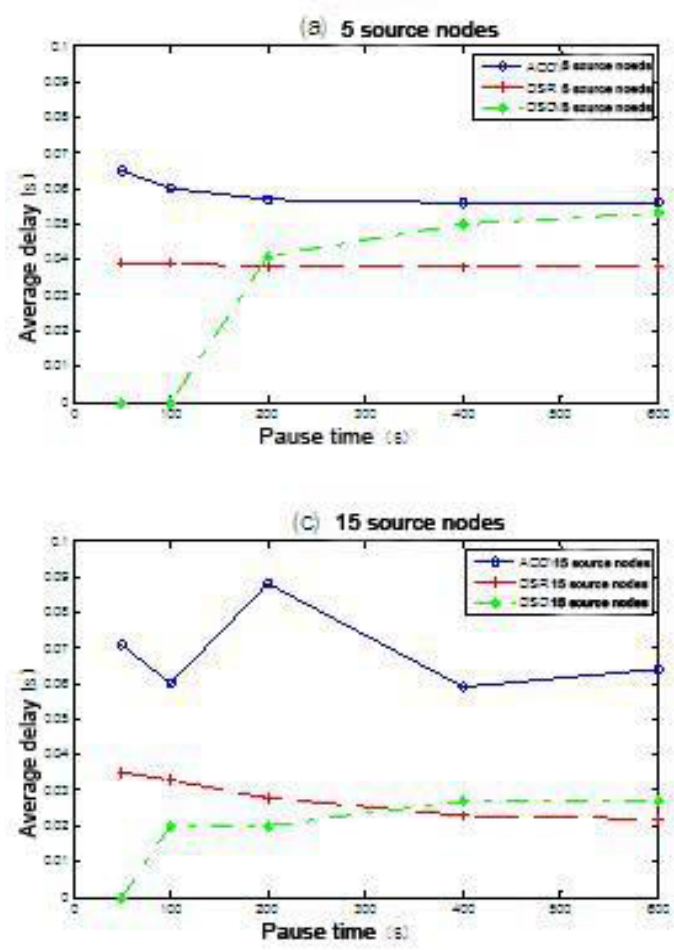

different trends: the delay of DSDV increase was relatively large and more than DSR. The delay of DSR slightly decreased, remaining flat basically, and the delay of AODV also had a certain degree of decline. The delay variation range of the three routing protocols was $0.05 \mathrm{~s}$ or so, and the difference between the two was not more than $0.08 \mathrm{~s}$. At the same time, with the increase of the number of source nodes, the overall change trend of DSR and DSDV routing protocols would increased. It was that the delay time would be much smaller, and the delay of AODV protocol increased slightly. But generally speaking, the delay performance of the three protocols was almost the same, and the biggest difference would be less than $0.08 \mathrm{~s}$.

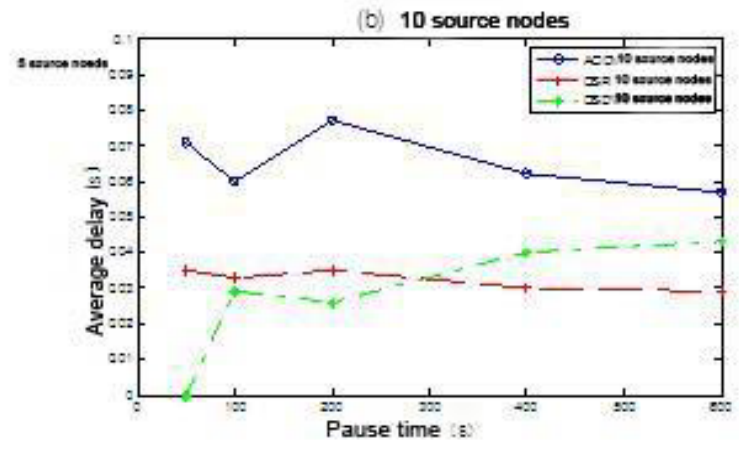

20 menos node

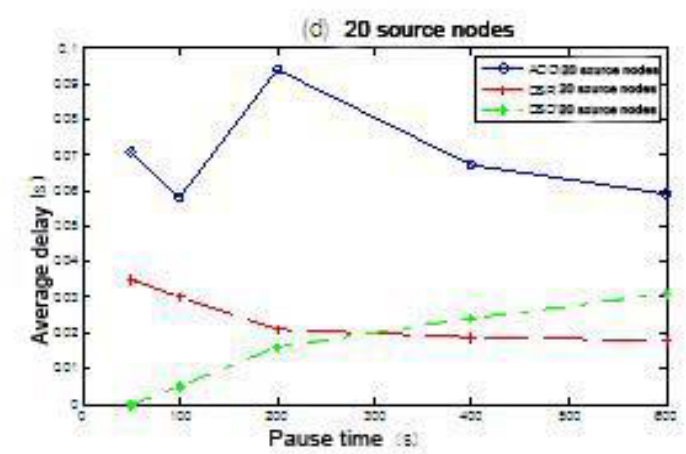

Figure 1.the average end-to-end delay performance of AODV、DSR、DSDV 


\subsection{Packet loss rate}

Packet loss rate refers to the ratio of the packet loss and the total amount of data packets. In the same scene, the packet loss rate of the vehicular ad hoc networks with AODV, DSR and DSDV routing protocols are simulated. In the scene of $5,10,15$ and 20 communication source nodes, through the simulation contrast experiment, the packet loss rate could be obtained with the increase of pause time and source nodes of the three routing protocols. The statistical results were shown in Table 1 and figure 1 .

Table 2. the packet loss rate of AODV、DSR、DSDV

\begin{tabular}{|c|c|c|c|c|c|c|c|c|c|c|c|c|c|c|c|}
\hline pause time & \multicolumn{3}{|c|}{$50 \mathrm{~s}$} & \multicolumn{3}{|c|}{$100 \mathrm{~s}$} & \multicolumn{3}{|c|}{$200 \mathrm{~s}$} & \multicolumn{3}{|c|}{$400 \mathrm{~s}$} & \multicolumn{3}{|c|}{$600 \mathrm{~s}$} \\
\hline $\begin{array}{l}\text { source } \\
\text { ode }\end{array}$ & AODV & DSR & DSDV & AODV & DSR & DSDV & AODV & DSR & DSDV & AODV & DSR & DSDV & AODV & DSR & DSDV \\
\hline 5 & 0.1602 & 0.7607 & 1 & 0.1649 & 0.7552 & 1 & 0.1574 & 0.7584 & 0.9368 & 0.1608 & 0.7558 & 0.6537 & 0.1630 & 0.7549 & 0.4967 \\
\hline 10 & 0.2407 & 0.7341 & 1 & 0.2860 & 0.7410 & 0.9984 & 0.3980 & 0.7215 & 0.8343 & 0.4410 & 0.7129 & 0.6765 & 0.4558 & 0.7131 & 0.6154 \\
\hline 15 & 0.2407 & 0.7341 & 1 & 0.2817 & 0.7306 & 0.9962 & 0.4451 & 0.6722 & 0.7666 & 0.4917 & 0.6404 & 0.671 & 0.5108 & 0.6227 & 0.671 \\
\hline 20 & 0.2407 & 0.7341 & 1 & 0.3143 & 0.7410 & 0.9759 & 0.4712 & 0.6752 & 0.7568 & 0.5410 & 0.6473 & 0.6835 & 0.5698 & 0.6404 & 0.6725 \\
\hline
\end{tabular}

As can be seen from Figure 2, the packet loss rate of AODV was kept at a relatively low level. With the increase of the number of source nodes, packet loss rate of AODV presented an upward trend, which was mainly due to a large number of traffic flow leading to network congestion, but the packet loss rate was still lower than DSR and DSDV. With the increase of the pause time and the number of source nodes, the packet loss rate of DSR

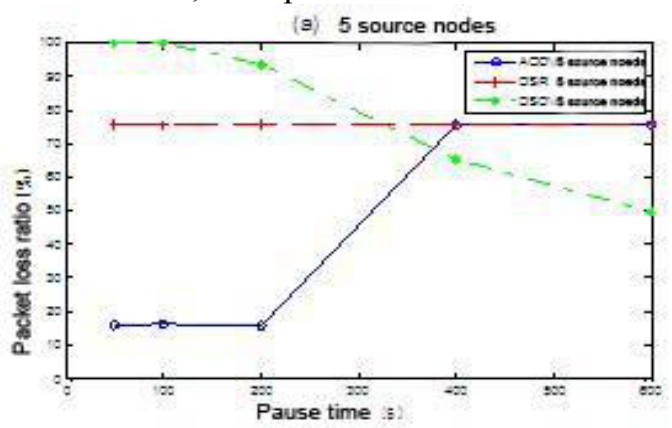

(c) 15 source nodes

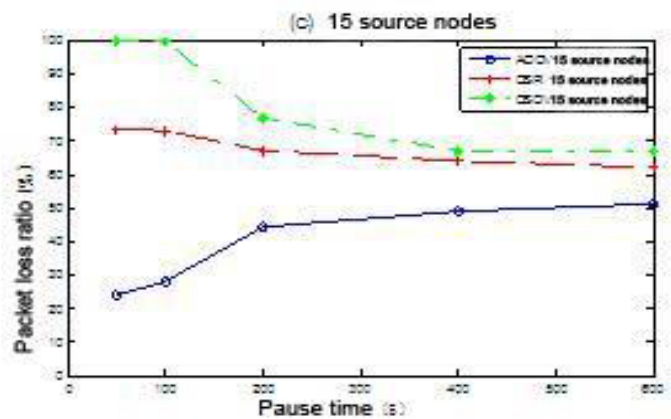

changed less, maintaining at about $70 \%$. In the case of short pause time, packet loss rate of DSDV was almost $100 \%$. With the increase of the pause time, packet loss rate of DSDV reduced to some extent. In addition, with the increase of the number of source nodes, the downward trend tended to relief. However, when the number of source nodes was more than 15 , packet loss rate of DSDV was always greater than AODV and DSR.
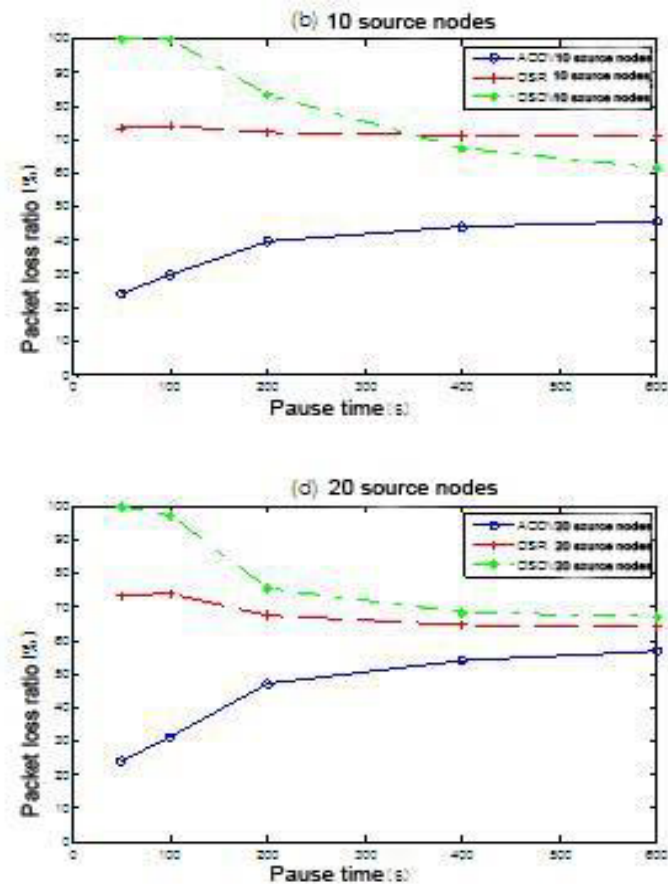

Figure 2 the packet loss rate of AODV、DSR、DSDV 
Through the above simulation results on the end-to-end delay and packet loss rate of the vehicular ad hoc network, the conclusion could be summarized: With the increase of the pause time and the number of source nodes, the delay of the AODV protocol was higher than the other two protocols at the same speed, but the maximum difference was not more than $0.08 \mathrm{~s}$. In terms of packet loss rate, packet loss rate of AODV was significantly lower than DSR and DSDV, showing a better ability to deal with network congestion. In the process of the moving vehicles, because of the high security requirements of the linear vehicles model, the information and the instructions should be transmitted to an optimal degree, and the delay did not need to be too harsh. Based on the performance of the end-to-end delay and packet loss rate of the vehicles linear model, the AODV routing protocol was suitable for the vehicular ad hoc network which maintained at a steady cruising speed.

\section{References}

[1] DUAN Yunfei, ZHOULiqiong , YANGLei. Simulation and analysis of several typical hoc Ad routing protocols $[\mathrm{J}]$. ]communication of electric power system , 2009,30(200): 59-63.

[2] CHENLinxin, ZENGXi , CAOYi. Mobile Hoc Ad network: self organizing packet radio network technology [M] . Beijing : Publishing House of Electronics Industry, 2012.

[3] Luo Pei'en, Huang Hongyu, Shu Wei, Li Minglu, Wu Minyou. Performance Evaluation of Vehicular DTN Routing under Realistic Mobility Models [C]. In: Proceedings of IEEE Wireless Communications and Networking Conference, 2008: 2206-2211.

[4] Ali S, Bilal S. An Intelligent Routing Protocol for VANETs in City

\section{SUMMARY}

VANET was known for the characteristics of autonomy, non-fixed structure, multi-hop routing, dynamic change of network topology, limited network capacity and so on. Meanwhile the application environment of VANET is very easy to meet the special circumstances, such as the narrow road, high density node distribution, high speed mobile of node and so on. For the above reasons, the transmission capacity of VANET would reduce, which makes the packet loss increases and the delay increases. The simulation experiments showed that with the increase of the pause time and the number of source nodes, the delay of AODV was a little higher than DSR and DSDV. But the biggest difference would be less than 0.08s. And AODV packet loss rate is significantly lower than DSR and DSDV, showing a better ability to deal with network congestion. 2nd International Conference on Computer, Control and Communication (IC42009), Karachi, Pakistan, 2009.

[5] Okada H, Takano A, Mase K. A Proposal of Linkmetric for Next-hop Forwarding Methods in Vehicular Ad Hoc Networks [C]. Proceedings of 6th IEEE Consumer Communication Sand Networking Conference, CCNC, Las Vegas, USA, 2009.

[6] Sommer C , Dressler F. Progressing Towards Realistic Mobility Models in VANET Simulations [J]. IEEE Communications Magazine, 2008, 46(11): 132-137.

[7] Hartenstein H, Laberteaux KP. A Tutorial Survey on Vehicular Ad Hoc Networks [J]. IEEE Communications Magazine, 2008, 46(6): 164-171. 